\title{
Hypervideo Design and Support for Contextualized Learning
}

\author{
Teresa Chambel \\ University of Lisbon \\ Portugal \\ tc@di.fc.ul.pt
}

\author{
Carmen Zahn \\ KMRC Tübingen \\ Germany \\ c.zahn@iwm-kmrc.de
}

\author{
Matthias Finke \\ CGC Darmstadt \\ Germany \\ matthias.finke@zgdv.de
}

\begin{abstract}
The aim of this paper is to discuss how new video based technologies, such as hypervideo, can provide authentic contexts to support meaningful learning. We approach the issue from both cognitive-psychological and technological perspectives. First, we discuss how cognitive and socio-cognitive processes generally involved in learning and collaborative knowledge building relate to the specific characteristics of hyperlinked videos, informing their design. Then, with regard to technology, we introduce two concepts, which provide tools for crafting and interacting with non-linear knowledge structures based on dynamic video information. Case studies and first research findings are presented and prospects for future research are outlined.
\end{abstract}

\section{Introduction}

New technologies do not only meet existing needs in terms of communication and learning practice, they can also redefine our educational culture by enabling new learning experiences in resource-rich learning environments [1]. For example, hypervideo, which refers to the integration of video in hypermedia structures, can be expected to contribute to learning in very distinct ways: as a presentation medium, it can support self-regulated cognitive processing of dynamic visualizations; as a non-linear and interactive medium, it allows for reflective and elaborative knowledge acquisition, individually or in groups $[3,7,24,25]$. These ideas will be discussed in more detail in the following sections.

\section{What is "hypervideo"?}

The term "hypervideo" reflects the idea of true integration of video in hypermedia spaces, where it is not regarded as a mere illustration, but can also be structured through links defined by spatial and temporal dimensions [2,3]. Hypervideo structures may as well be defined as a combination of interactive video and hypertext, as they consist of interconnected video scenes, that may further be linked with additional information elements, such as text, photos, graphics, audio or other videos in the hypermedia space [23].

The roots of hypervideo structures lie in the early days of hypertext, when Ted Nelson extended his hypermedia model to include "branching movies" or "hyperfilms" [12]. However, technology has been slow in bringing these ideas to full realization [2,3]. HyperCafe [19] is one of the earliest hypervideos, featuring digital video and revisiting hypermedia concepts in this scenario. Since then, different types and levels of video integration in hypermedia have evolved [23]. For example, we might differentiate between (1) homogeneous hypervideo, where video is the only medium involved, consisting of dynamic audio-visual information presented as a continuous stream of moving pictures that can be navigated by the user, and (2) heterogeneous hypervideo that integrates other media, providing further and related information to the video, or having video illustrate and complement it $[23,3]$. For this broader perspective, which we will focus on in this paper, the name of "video based hypermedia" or "hyperlinked video" is sometimes adopted [3,25].

\section{Learning with hypervideo}

An effective design of tools and environments that support learning requires the understanding of human cognition and learning processes. This section presents the main cognitive concepts relevant for discussing video and hypervideo as supporting tools for learning.

3.1. Cognitive modes, learning phases and learning styles 
Norman [14] identifies two cognitive modes: the experiential mode relates to a state in which we perceive and react to events in an effortless way, it is about perception and motivation, and good for accretion of facts and tuning of skills; while the reflective mode relates to comparison and contrast, thought and decision making, essential for restructuring of knowledge. Both are important in human cognition, but they require different kinds of technological support.

In addition to cognitive modes, different Learning Phases have been identified for the learning process. The classic learner centered pedagogy model has three phases: (1) conceptualization of the subject and its domain; (2) construction, where the learner actively engages with the subject, while relating to her own knowledge framework; and (3) dialogue, where the learner expresses aspects of the emerging understanding and relates this to the understandings of fellow learners and tutors.

Besides different cognitive modes and learning phases in individual learning, people also develop different Learning Styles, or cognitive preferences, that determine their best suited ways to learn. There are many theories, models, and instruments to determine learning styles, but they are all essentially based on the idea that individuals perceive, organize or process information differently. Examples of these theories include: the VAK Perceptual Learning Styles: visual, auditory, and kinesthetic; and the Kolb's Learning Styles Inventory [8], identifying four different styles: reflector, pragmatist, theorist, and activist. This differentiation suggests a need for a flexible support of different styles. An ideal learning environment would support all the learning styles, with the flexibility to allow each learner to spend more time on her preferred style, and induce the development of skills in non-dominant styles.

\subsection{Video as a cognitive tool}

In some learning situations, videos or animations are not only a desirable, but a necessary prerequisite for successful learning, for a number of topics or problems exist that can hardly be understood without using dynamic visual materials as a referential basis. From a cognitive perspective, audiovisual materials support learning (1) by 'replacing' real experience, because of their authenticity and realism, which evoke feelings of "observing real situations" [20], (2) by visualizing dynamic processes, which might not be observable in reality or which are hard to describe verbally [16] and (3) by combining diverse symbol systems, such as pictures, texts and narration, into coherent media messages [11]. The specific qualities of video presentations are supposed to support the construction of rich mental representations and, by dual coding [15,11] improve the transfer of knowledge. In accordance with these assumptions, empirical findings have consistently shown that dynamic media facilitate the comprehension and transfer of knowledge in individual learning [16,11].

In specific collaborative scenarios, video can as well be considered supportive for cognitive processing. For reasons similar to those in the case of individual learning, video is helpful when meaningful collaboration depends on visual perceptions of concrete objects, actions, or complex relations; and when knowledge is created within networked groups, where learners do not meet in the same place at the same time and, hence, cannot observe the same things in the same situation.

To summarize, video, as dynamic and figurative information combined with verbal audio, forms a powerful means of communicating meaning-scenarios rapidly and efficiently $[10,15]$. It can bring context to topics and enhance the authenticity of a computer based learning environment, thus fostering what Norman [14] called an experiential cognitive mode. However, to allow reflection, a system must have a medium that affords adding, modifying and manipulating representations, and performing comparisons. It must also afford time for reflection, elaboration, and comparison processes. Broadcast television, and most videos, are usually watched in an experiential mode, and cannot augment human reflection in this sense [14]. According to Eco [6], the lack of communication and debate is also an important drawback in television teaching capabilities. He defends that learning should take place in a broader context where discussion could happen.

\subsection{The role of hypervideo}

Television and video could as well be a powerful tool for reflection, if designed in a way that would allow the viewer to select what to watch, to control the pace of the information flow [14,23], to stop and make annotations [5], and to relate to other materials [14] or to other people's points of view.

Hypervideo technology can provide this kind of support to the different learning modes, phases and styles, and integrate an environment that allows the communication and collaboration among learners, teachers, and other experts on the subjects at hand $[3,5,7,23,25]$. Hypervideo might promote learning in 
its main modes and phases, for different individuals (see section 3 ) by providing and supporting:

1) Interactive access to rich audio-visual information, contextualized in video based hypermedia spaces.

2) Construction of knowledge in a sense of relating concepts and expanding on them, using annotations and cognitive maps.

3) Communication, allowing the debate of ideas, the exchange and sharing of information and knowledge, or the collaborative elaboration of the previous hypermedia spaces.

By allowing the viewer to watch video in its natural experiential mode, inducing and supporting more active and reflective attitudes, through control, comparison, and annotations, hypervideo can support both cognitive modes suggested by Norman [14]. Its ability to integrate heterogeneous media and activities also allows the support of diverse learning styles. However, all this power goes along with potential complexity and may not be used to its best in all cases, especially for learners and authors with little experience and background knowledge. Hence, design guidelines play an essential role in the authoring of effective hypervideo spaces [3,23].

\section{Design for learning with hypervideo}

Hypervideo shares with traditional hypermedia the potential of increased cognitive load that might also lead to disorientation $[4,13]$. But, in the case of hypervideo, this kind of problem might be even more pronounced, because, for its richness, the video itself sometimes carries with it the risk of overstraining the cognitive capacities of the learner, and the dynamic nature of nodes and links may put time pressure on the users, when they are required to make navigational decisions [23]. The integration of a dynamic medium, like video, with static media, such as text and images, also raises important rhetorical and aesthetic challenges to hypervideo, since they induce different attitudes in the user [9,19,3].

Hence, the effective design of hypervideo spaces may greatly benefit from following guidelines, that help to face the extra challenges video brings to the scenario, and an adequate support from the underlying systems, that bring these hyperspaces to life, as exemplified in the next two sections. Many of the mechanisms adopted for hypermedia need to be extended for hypervideo, in order to accommodate its increased complexity, and should address the provision of [3]: control, to be able to navigate the videos and the hyperspace, for example, extra mechanisms have to be available in order to provide users with information about the existence (where, when, for how long) of links on the video - 'link awareness' [2], a more complex issue in hypervideo, since video changes in time; consistency and coherence, to reduce cognitive load [23]; context for orientation purposes, e.g. through the synchronization of video with navigation maps; and familiarity, for example through the adoption of metaphors, like television, books, and traveling; and continuity, especially when navigating between dynamic and static media, for a sense of unity and coherence.

In collaborative situations, the demanding nature of multimedia-based design activities may raise a series of problems, hindering learning effects. For example, students may feel overwhelmed, that a greater focus on design and production tasks may distract them from knowledge content, and that the group may have difficulties with the organization of its work $[17,18]$. Especially when working with videos, such problems might well lead to a situation, which reduces the capabilities and motivations to engage in meaningful collaboration.

Thus, it appears a basic necessity to think carefully about how to adapt technology to the cognitive prerequisites of potential hypervideo users, thereby setting the groundwork for effective and satisfying learning to take place. The design approaches specified in the next paragraphs encompass (1) information is mainly presented by audiovisual media, (2) knowledge can be created collaboratively on the basis of video presentations, by means of both linking information and annotating, and (3) the process of knowledge building is reflected in resulting visualizations in the hypervideo structure.

\section{Hypervideo in HTIMEL}

To explore the use of hypervideo in learning environments, we developed the HTIMEL (HTML with Time Extensions) model and language, as an extension to HTML and existing Web tools [2]. The temporal dimension was considered, to allow the addressing of video in space and time, for the definition of link anchors, and to synchronize media elements. New forms of integration, annotation, and navigation of video in hypermedia were conceived, with a special concern to the support of cognitive processes. These tools were used to create course material, mainly in Literature and Mathematics, following our ideas about the way video should be integrated and augmented to support learning [3]. Case studies were developed in collaboration with the 
Portuguese Open University, in the context of distance learning, and in collaborations with the Center for Mathematics and Fundamental Applications at the University of Lisbon, CalTech, and The Technical University of Berlin.

The example presented in fig.1 illustrates one of our case studies. The information is organized around text based and video based pages, that closely relate to the book and videotapes that were originally used in the course. Through their integration in hypervideo, relations among them could be fully captured, maintaining the original context. In text centered pages, video is integrated as an illustration that is played when the hypervideo links defined in the text are followed. The user has an augmented experience of text reading.

The user may focus on the videos, through video centered pages, where the whole video can be played. Indexes, synchronized with the video, make the video structure explicit, provide for user orientation, and can be used to navigate the video, providing control to the user. Different types of indexes act as different views or maps of the video. For example, a table of contents (fig.1b) represents the video structure, whereas an image map (fig. $1 \mathrm{c}-\mathrm{d}$ ) is a visual summary of the video; a cognitive map represents its knowledge structure [7], while user annotations [5] capture a personal view of the video, and exercise maps [3] relate activities and quests to video content. In video centered pages, the user has an augmented experience of video watching.

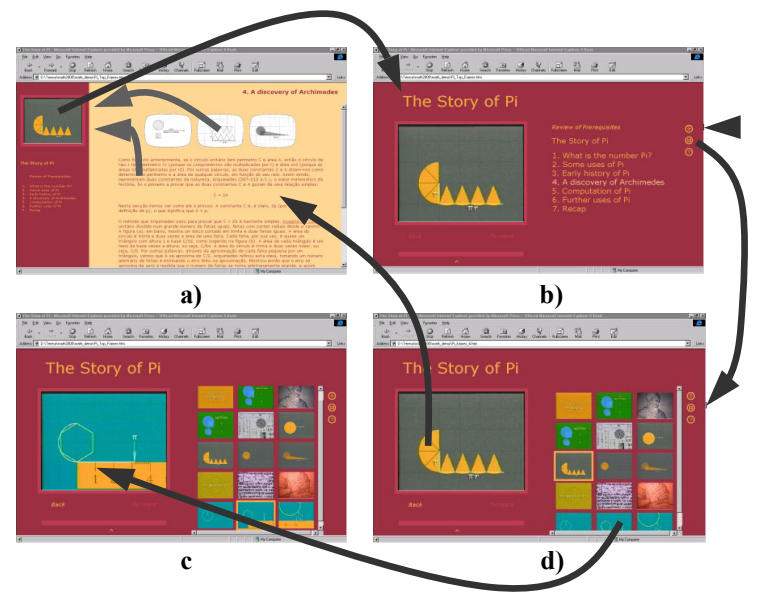

Figure 1. Hypervideo example in HTIMEL

Links can also be defined among different parts of the video, addressed in space and time, allowing to navigate it through related information, or among portions of video and text (fig.1d-a), complementing or contextualizing the information conveyed by each of them. Being developed for the Web, these hypermedia spaces allow an easy integration of communication mechanisms. Students may then create their own versions of hypervideo documents, expand upon the original ones, through annotation, and share them with colleagues and teachers.

From our studies and experiences, we conclude that students are more motivated to watch the videos in this type of hypervideo, than in traditional settings, as the process becomes more flexible and engaging; and it is easier to search for information and to capture the videos' messages through the different maps available. Video course material, as rich as it is, is better used in a reflective mode if presented in a way where the content or knowledge structure is made explicit. The full integration of video in hypermedia also allows the capturing of important relations between video and other media, like text, through contextualized explanations and illustrations that promote deeper understandings of the different materials. Different learning styles are also supported, through the integration of different media and perceptual modalities, interaction, and navigation choices $[3,7]$.

\section{Dynamic Information Spaces (DIS)}

To explore the use of hypervideos in collaborative learning scenarios the Hypervideo System presented in the following paragraphs was developed. Here, hypervideo is conceptualized as a complex dynamic information space ("DIS"), where learners may add their own materials to selected video objects and make specific annotations, while collaboratively expanding their knowledge on the topic at hand. The general approach is aligned with considerations of cognitive psychology and theories of CSCL [22]. It is thus combining (1) interactive presentation processes with (2) collaborative editing processes as the basic design concepts and core functionalities of the system (for details see [25]).

The system architecture is based on the World Wide Web as the largest accessible hypermedia system for public use that provides the needed connectivity and data transfer features. The architecture follows a modular concept. Since the modules are designed as independent units, changes to one module cause a minimum influence on the other modules [25]. In order to avoid inconsistency in the dynamic information space due to user contributions, the system design is based on a client-server model. An example of the user-interface, for the learners to add their contributions and annotations, is shown in figure 2. It was developed for our experimental work and case studies in the context of university teaching. The 
experimental research conducted at the University of Tübingen/Germany in cooperation with the Computer Graphics Center Darmstadt/ Germany with an earlier version of this Hypervideo System revealed that hypervideos provide a very successful mode of learning, also positively acknowledged by the satisfaction of the subjects $(\mathrm{N}=74)$. Results further suggest that slight variations in design options concerning position and number of links to additional information did not significantly influence navigation and successful knowledge acquisition in individual learning, but individual strategies and navigation behaviour, including exploration activity, redundancy, and duration of links activated, were significantly and positively correlated with knowledge acquisition [24].

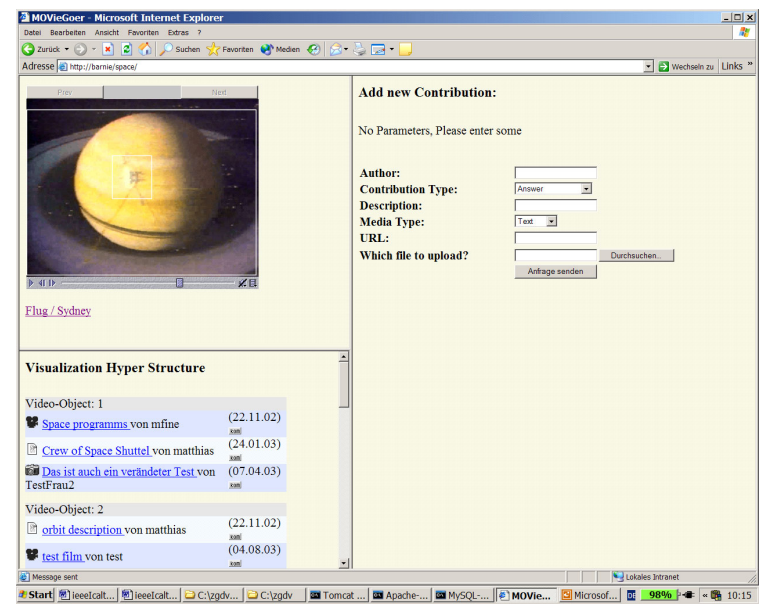

Figure 2. Example of the Hypervideo System

Our current research projects focus on collaborative learning and include an implementation study with two media psychology courses at the Universities of Muenster/Germany and Linz/Austria. In this project, about 30 advanced psychology students collaboratively designed hypervideo structures on "presentation techniques". First results indicate a great general satisfaction with the work of crafting hypervideos with the system. Further paramount questions in this study concentrate on the students' communication patterns, on collaborative writing processes and on the groups' products. Results can be reported in more detail, soon.

\section{Conclusions and Perspectives}

From a cognitive viewpoint, video can be considered a powerful referential anchor, serving to stimulate and facilitate both individual and collaborative processes of learning and knowledge building, particularly in specific domains, such as the natural sciences, which rely heavily on visual phenomena. Video provides context information in an efficient way but has limited advantages to reflective learning. Hypervideo additionally allows more control and the composition of rich and flexible knowledge structures, corresponding to enriched mental models. Learners may also express their internal knowledge structures externally and share and discuss them with other students. Thus, hypervideo provides a better support to reflection and learning, in accordance with learners' individual needs and styles, and different learning phases $[3,7,23]$.

From our experiences, we concluded that the ability to integrate video in rich hypermedia spaces enables learners to create rich representations and promotes deeper understandings. It improves both text and video understanding, due to the contextualized explanations made possible by the integration of both materials. Important relations with video information can be captured. However, there are some main challenges, including the management of potentially high cognitive load in processing information based on hypervideo, and usually significant investments, in time and money, involved in the production of multimedia and video based products. The definition of design guidelines and the development of effective and flexible production frameworks and authoring tools can help to meet these challenges. Some work has been done in this direction $[2,3,23,24]$, but more research needs to be done. We intend to explore a broader approach to the support of individual and collaborative learning, inspired by field studies in different learning contexts that might raise specific challenges and require differentiated support.

\section{References}

[1] Beichner, R., "Multimedia editing to promote science learning", Journal of Educational Multimedia and Hypermedia 3 (1), 1994, pp.55-70.

[2] Chambel, T., N. Correia, and N. Guimarães, "Hypervideo on the Web: Models and Techniques for Video Integration", International Journal of Computers \& Applications, Acta Press, Vol. 23, \#2, 2001,pp.90-98. ISSN: 1206-212X

[3] Chambel, T., and N. Guimarães, "Context Perception in Video-Based Hypermedia Spaces", in Proceedings of ACM Hypertext'02, College Park, Maryland, USA, 11-15 June 2002.

[4] Conklin, J., "Hypertext: A Introduction and Survey", In IEEE Computers, Vol.20, 1987, pp.17-41. 
[5] Correia, N., and T. Chambel, "Active Video Watching Using Annotation", In Proceedings of ACM Multimedia'99, Orlando, Florida, USA, November 1999.

[6] Eco, U., "Can Television Teach?", Screen Education, no.31, Summer 1979 .

[7] Guimarães, N., T. Chambel, and J. Bidarra, "From Cognitive Maps to Hypervideo: Supporting Flexible and Rich Learner-Centred Environments", IMEJ-Interactive Multimedia Electronic Journal of Computer-Enhanced Learning, Volume 2, Number 2, Oct. 2000. ISSN: 1525-9102.

[8] Kolb, D., Experiential Learning: Experience as the Source of Learning and Development, Englewood Cliffs: Prentice Hall, 1984.

[9] LiestØ1, G., "Aesthetic and Rhetorical Aspects of Linking Video in Hypermedia", In Proceedings of ACM Hypertext'94. Edinburgh, UK, 1994, pp.217-223.

[10] LiestØl, G., Essays in Rhetorics of Hypermedia Design, $\mathrm{PhD}$ Thesis, Department of Media \& Communication, University of Oslo, Norway, 1999.

[11] Mayer, R., Multimedia Learning. Cambridge: Cambridge University Press, 2001.

[12] Nelson, T., "Branching presentational systemsHypermedia”, Dream Machines, 1974, pp.44-45.

[13] Nielsen, J., Multimedia and Hypertext - The Internet and Beyond, Academic Press Inc., 1995.

[14] Norman, D., Things That Make Us Smart, Addison Wesley Publishing Company, 1993.

[15] Paivio, A., Mental representation: A dual coding approach, Oxford, England: Oxford University Press, 1986.

[16] Park, O., and R. Hopkins, "Instructional Conditions for Using Dynamic Visual Displays: A Review", Instructional Science, 21, 1993, 427-448.

[17] Posner, I., R. Baecker, S. Poplar, and M. Becker, "Children Collaborating in Making Movies Using Computer Based Multimedia: Multimedia Summer Camp Revisited", poster session presented at the Computer Supported Collaborative Learning (CSCL) Conference, 1997.
[18] Reinmann, P., and J. Zumbach, "Design, Diskurs und Reflexion als zentrale Elemente virtueller Seminare" (Design, discourse, and reflexion as central elements of virtual seminars), In F. Hesse, and H. Friedrich (eds), Partizipation und Interaktion im virtuellen Seminar, Münster: Waxmann, 2001, pp.135-163.

[19] Sawhney, N., D. Balcom, I. Smith, "HyperCafe: Narrative and Aesthetic Properties of Hipervideo", In Proceedings of ACM Conference on Hypertext'96, Bethesda, MD, 1996, pp.1-10.

[20] Schwan, S., "Video in Multimedia-Anwendungen. Gestaltungsanforderungen aus kognitions-psychologischer Sicht“ In: Krampen, G., Zayer, H. (ed.). Psychologiedidaktik und Evaluation II: Neue Medien und Psychologiedidaktik in der Haupt- und Nebenfachaus-bildung. Bonn: Deutscher Psychologenverlag, 2000.

[21] Schwan, S., "Do it yourself? Interactive visualisations as cognitive tools". Learning and Instruction, in press.

[22] Stahl, G. "Contributions to a theoretical framework for CSCL. In: G. Stahl, (ed.). Computer Support for Collaborative Learning: Foundations for a CSCL Community - Proceedings of CSCL 2002 Boulder, Colorado, USA, January 7-11 2002. (pp. 72-81).Hillsdale, N.J., USA: Lawrence Erlbaum Associates, Inc.

[23] Zahn, C., Schwan, S., and Barquero, B. "Authoring hypervideos: Design for learning and learning by design", In: Bromme, \& Stahl, E., Writing Hypertext and Learning: Conceptual and Empirical Approaches. Oxford: Elsevier Science, 2002.

[24] Zahn, C., Barquero, B. and Schwan, S. "Learning with hyperlinked videos - design criteria and efficient strategies of using audiovisual hypermedia", Learning and Instruction, in press.

[25] Zahn, C., and Finke, M. "Collaborative knowledge building based on hyperlinked video", In B. Wasson, R. Baggetun, U. Hoppe, \& S. Ludvigsen (eds.), Proceedings of the International Conference on Computer Support for Collaborative Learning - CSCL 2003, COMMUNITY EVENTS - Communication and Interaction Bergen, NO: InterMedia, 2003, pp.173-175. 